\title{
Germanica
}

\section{Les univers intérieurs de Gertrud Kolmar : à propos de Welten (1937)}

Gertrud Kolmars Innenwelten : über den Gedichtzylus Welten (1937)

Gertrud Kolmar's inner universes: considering Welten (Worlds)(1937)

\section{Laurent Cassagnau}

\section{CpenEdition}

\section{Journals}

Édition électronique

URL : http://journals.openedition.org/germanica/248

DOI : 10.4000/germanica.248

ISSN : 2107-0784

\section{Éditeur}

Université de Lille

\section{Édition imprimée}

Date de publication : 1 juin 2007

Pagination : 103-121

ISBN : 2-913857-19-1

ISSN : 0984-2632

\section{Référence électronique}

Laurent Cassagnau, « Les univers intérieurs de Gertrud Kolmar : à propos de Welten (1937)»,

Germanica [En ligne], 40 | 2007, mis en ligne le 16 février 2010, consulté le 06 octobre 2020. URL

http://journals.openedition.org/germanica/248 ; DOI : https://doi.org/10.4000/germanica.248

Ce document a été généré automatiquement le 6 octobre 2020.

(c) Tous droits réservés 


\title{
Les univers intérieurs de Gertrud Kolmar : à propos de Welten (1937)
}

\author{
Gertrud Kolmars Innenwelten : über den Gedichtzylus Welten (1937) \\ Gertrud Kolmar's inner universes: considering Welten (Worlds)(1937)
}

\section{Laurent Cassagnau}

Un des plus beaux poèmes d'Ingeborg Bachmann porte comme titre « La Bohême est au bord de la mer» («Böhmen liegt am Meer») : s'appuyant sur le Conte d'hiver dont l'action se passe en partie en Bohême, que Shakespeare place au bord de la mer, I. Bachmann construit un espace utopique qui permet à la fois la perte du Moi et sa reconstruction :

Je ne désire plus rien. Je veux sombrer au fond.

Au fond - c'est-à-dire à la mer, j'y retrouve la Bohême.

Ayant touché le fond, je m'éveille sereine.

À présent, je sais qu'au fond je ne suis pas perdue.

Approchez, Bohémiens, et vous aussi, marins et catins des ports, navires

Sans amarres. Ne voulez-vous pas être Bohémiens, vous tous, Illyriens, Véronais

Et Vénitiens. Jouez les comédies qui font rire

Et qui sont à pleurer $[. . .]^{1}$

2 Peut-être I. Bachmann avait-elle à l'esprit, lorsqu'elle composa ce texte, le poème «Phares » (« Türme ») de Gertrud Kolmar paru dans le cycle Mondes (Welten), écrit en 1937 et publié par Peter Suhrkamp en 1947, après la mort de la poétesse en déportation ${ }^{2}$. Dans ce poème qui évoque quatre phares, placé chacun à quatre points cardinaux, la Bohême maritime de Shakespeare fait déjà figure d'ailleurs :

Quelque part en Bohême un bouleau incline ses étroites joues

baignées de blondeur sur des ruines rougeâtres.

[...]

des yeux luisant d'avidité des requins bleus qui, happant, aux aguets, s'ébrouent

dans les eaux côtières,

Rejaillit le regard des seigneurs de la forteresse, âme des pirates illyriens [...]

ô noirs pavillons, équipées de corsaires, abordages en armes contre les Vénitiens ${ }^{3}$ ! 
Mais si la Bohême de Bachmann marque une situation d'horizon à partir de laquelle s'enclenche pour le Moi une dialectique de l'ici et de l'ailleurs, de la perte et de la recréation ( «Je confine encore à un mot et à une autre terre/ Même peu, je confine à tout et toujours plus encore,/ Un Bohémien, un vagabond qui n'a rien, qui ne tient rien/ dont le seul don n'est plus que de voir, depuis la mer incertaine, la terre de mon choix »'), chez Kolmar la Bohême shakespearienne n'est qu'un souvenir, un intertexte dépossédé de vie, mais non de beauté :

Du passé.

Des pièces délabrées

Ne résonnent plus les timbales dorées d'ivresse qui sont remplies

d'un vin couleur de sang ${ }^{5}$

Le phare en ruine sur le rivage est envahi de lézards aux reflets de jade, la femelle du scorpion meurt en donnant la vie à sa progéniture, mais, comme il est dit, «les fils héritent du poison du père $»^{6}$. Cette tour, comme les autres évoquées dans le poème, se dresse dans un paysage qui, malgré sa beauté, est désolé. G. Kolmar semble reprendre dans l'ensemble du poème une typologie bien connue dans l'espace germanophone (et sans cesse actualisée de Nietzsche à Gottfried Benn) qui oppose le Nord au Sud et à l'Orient. Mais si le phare du Nord se dresse ici dans un paysage inhospitalier (« Sur la plage de la mer nordique/ Où la cruelle tempête noire fouette des nuées de mouettes [...] le phare se dresse./ Dur, sinistre, pesant, muet dans la triste grisaille »7), la tour du Sud semble, elle aussi, construite dans un paysage aride et stérile, écrasé de soleil :

Indiciblement vide, le ciel qui ne connaît pas plus l'aile de l'oiseau que

le nuage fécond, à l'infini sur lui déverse

un azur brûlant ;

Des chaleurs torrides, des flots rayonnants s'écoulent de ses livides boutisses8

5 Le poème s'achève sur le lien que le Moi lyrique parvient à établir, de nuit, avec cet ailleurs situé au Levant, c'est à dire à la fois au Sud et à l'Est, renvoi explicite aux « origines juives » de Kolmar :

Peut-être mon âme m'a-t-elle oubliée en rêve,

Déployée vers le Levant, elle piqua ; et le phare blanc

Attendait son vol changeant : à travers ses chaudes

pièces sans vie, enchantées, elle erra

À la recherche de ses ancêtres,

Et dans son essor effleura des cordes qui encore résonnent... ${ }^{9}$

6 On trouve ici le paradoxe d'un ailleurs à la fois refuge d'une âme en quête de ses ancêtres et lieu visible de la scission qui affecte le sujet dépossédé précisément de cette âme : la rencontre de l'âme et de la tour, si elle scelle l'union avec ses origines, désigne aussi l'aliénation du Moi. La construction de ce lieu lointain sert à évoquer la présence de l'âme sur le mode de la disparition, elle relève donc d'une forme de symbolisation, ce que confirme la métonymie des cordes qui renvoie à la lyre ou au psalterion biblique, donc au lyrisme. C'est en s'évanouissant que l'âme, par la musique qu'elle produit reste en contact avec le sujet qui ainsi la perçoit.

Ce poème figure dans Mondes, le cycle de 17 poèmes écrit à la fin de l'année 1937, soit deux ans après la promulgation des lois raciales de Nuremberg, six ans avant la mort de Gertrud Kolmar (de son vrai nom Gertrud Chodziesner) à Auschwitz. La construction de "mondes", d'univers imaginaires dans lesquels on ne trouve pas de références explicites à la persécution antisémite au moment même où l'administration nazie met en place des mesures de plus en plus répressives et discriminatoires apparaît comme 
une forme de fuite hors de la condition faite à Kolmar. Peut-on dire cependant que Kolmar « se réfugie » dans un univers irréel de création littéraire ? Il est indéniable que les poèmes de Mondes portent pour la plupart la marque d'un ailleurs à la fois spatial et temporel, littéraire et biblique, occidental et « oriental » (exotique), le pluriel du titre suggérant d'emblée que les poèmes vont mener le lecteur au-delà de la singularité, voire de l'étroitesse de ce monde-ci. De la licorne qui, dans le poème du même nom, se déplace d'Inde en Arabie, il est dit qu'elle vient d'Ophir, port mentionné dans l'Ancien Testament, d'où la Reine de Saba aurait fait envoyer des cargaisons d'or au Roi Salomon et que l'archéologue allemand Karl Gottlieb Mauch, à la fin du XIX siècle, avait cru retrouver lors de fouilles menées au Zimbabwe. Le poème retrace la course de l'animal fabuleux, qui le mène des forêts indiennes jusqu'aux déserts d'Arabie et s'achève sur la rencontre avec une figure féminine :

Passée la licorne, passée.

Car ses pas légers et rapides venaient de très loin, du

pays de l'or, Ophir,

[...]

Pourtant la corne oblique au milieu de son front diffusait une lumière

plus douce aux reflets laiteux

Sur les mains nues et les seins mollement voilés

de la femme $[. . .]^{10}$

8 Si ce poème, qui présente entre autres des réminiscences rilkéennes, débouche sur une rencontre aux connotations sexuelles, l'ailleurs oriental traversé par l'animal fabuleux n'apparaît pas sous les traits stéréotypés d'un exotisme sensuel et opulent, mais comme un paysage désolé vidé de ses habitants :

Elle vit

Des villes solitaires, livides, leurs coupoles et leurs minarets et les pierres des

champs funéraires,

Muettes sous le tintement de la lune.

Elle vit

Des ruines, des lieux abandonnés, habités par les seuls esprits,

dans les ténèbres étincelantes

Sous des astres froids.

Une fois la chevêche appela,

Et au loin les chacals hurlèrent leur plainte ;

Des hyènes rirent ${ }^{11}$.

Les espaces créés par Kolmar dans ces 17 poèmes, s'ils comportent des éléments exotiques et esthétisants, ont partie liée avec la mort. La fuite devant une réalité politique de plus en plus oppressante et porteuse de mort donne lieu, au sens propre du terme, à des univers marqués eux aussi par l'angoisse et la mort. L'écriture apparaît alors non pas comme une simple conduite de fuite hors de la vie, mais comme anticipation d'une mort dans l'espace littéraire, ou pour le dire avec le titre d'un des poèmes, d'une disparition dans l'espace de l'« art ».

Les poèmes du cycle, comme on le verra, jouent sur le double mouvement d'extériorisation et d'intériorisation, ce qui empêche le poème de se faire simple " escapisme » : l'espace recherché ou construit n'est pas que pure extériorité, c'est la raison pour laquelle il ne se réduit pas à l'exotisme d'un paysage pittoresque.

Il est vrai que Kolmar s'est intéressée aux cultures asiatiques, allant même jusqu'à se définir dans une lettre de 1939 comme une " asiatique contrariée ». Le poème "Asie " du cycle Mondes témoigne également de cette identification. Plus particulièrement, ce 
que Kolmar semble retenir de l'Asie est l'idéal d'impassibilité, le dépassement de l'activité et de la volonté dans la quête de l'Être. Il est donc intéressant d'analyser si les poèmes de Kolmar arrivent à se constituer et à se fixer en un paysage (exotique ou non) qui serait une figuration de cette utopie de l'Être, alors qu'à l'extérieur, dans le Berlin nazi, se déchaîne un vouloir-faire, pour ne pas dire une " volonté de puissance ».

Ceci est d'autant plus nécessaire que, dans le cas du cycle Mondes, le lecteur est confronté à un problème d'édition qui a des incidences sur la signification même de l'ailleurs. Dans son étude ${ }^{12}$, Ludwig Völker a montré combien l'édition de Hermann Kasack était problématique : faisant fi du tapuscrit et de la numérotation des poèmes de la main même de l'auteur, H. Kasack a placé en première position le poème "Art ", qu'il a rebaptisé « Dédicace » ("Zueignung ») et a mis en dernière place le poème "Asie » où le continent asiatique apparaît comme le lieu de la nostalgie. De la sorte, le poème " Art » acquiert un statut de programme liminaire et le poème « Asie » apparaît comme telos du cycle : le recueil convergerait vers ce poème final dans lequel est évoqué en détail un ailleurs situé à l'Extrême-Orient. Or dans l'agencement choisi par Kolmar ellemême, le cycle commence par " Les îles Mergui » : le motif asiatique est donné d'emblée au même titre que d'autres. Cela est confirmé par la position en avant-dernière place du poème "La Ville" qui évoque sans ambiguïté le port de Hambourg. Le poème "Asie ", lui, est placé très exactement au centre (en neuvième position) et sert d'axe ou de pivot au cycle qui débouche sur le poème "Art ». L'ordonnancement du cycle a donc été soigneusement pensé. Comme le montre l'analyse de Ludwig Völker, l'art, ou plus exactement l'entrée dans l'espace de l'art n'est pas posée comme préalable au cycle, mais comme but. Or dans ce poème final est décrite, en termes qui évoquent une expérience proche de la mystique, la façon dont une jeune femme, arrachée à son entourage qui ne comprend pas ses dessins, se trouve transportée par un nuage jusqu'à un personnage qui l'attend et la salue :

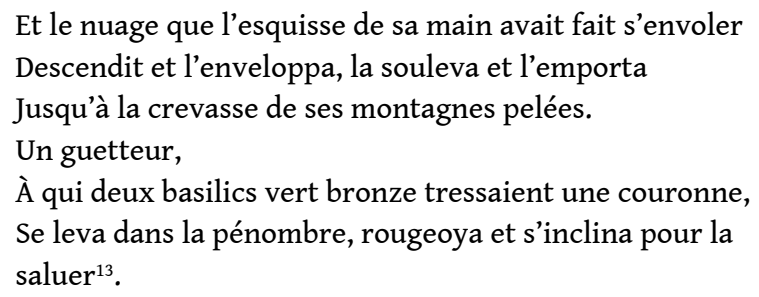

13 La figure qui se tient sur le seuil de l'espace fictionnel est couronnée de deux basilics, c'est-à-dire de serpents fabuleux dont le regard pétrifie. Le passage de la vision transposée en dessin à la négation de la vision, du geste du sujet créateur à la splendeur pétrifiante de celui qui se tient dans une muette attente est caractéristique du recueil. Les univers évoqués dans ce recueil apparaissent comme des mondes intérieurs qui résultent d'une biffure de la représentation graphique extérieure, y compris dans ce qu'elle a d'imaginaire et de poétique. Ce moment de refus sert d'élément structurel à plusieurs poèmes du cycle. «Art » commence par le geste du Moi poétique qui dessine au crayon un paysage : «Elle prit le crayon à pointe d'argent/ Et lui ordonna de glisser sur la blanche surface mate, son pays $»^{14}$. Or ce n'est pas cette première esquisse qui déclenche le moment épiphanique, mais la douleur face à l'incompréhension des autres et à leurs commentaires : "Sans couleurs, sans âme, sans voix est tout cela qui ne nous parle pas. Passez votre chemin $»^{15}$ s'exclament-ils devant le dessin qui représente des montagnes désertiques aux arêtes rocheuses. Se produit alors une rupture dans l'expérience, une fermeture au monde du visible comme en témoignent les réactions du 
corps ( Seule son épaule tressaillit, son regard fondit en larmes $\left.»^{16}\right)$. Sur le modèle de la célèbre légende chinoise du peintre disparaissant dans le paysage qu'il vient de peindre (légende que G. Kolmar devait connaître de ses lectures consacrées à l'ExtrêmeOrient) le sujet sort de lui-même et entre dans l'espace du dessin : il se voit transporté jusqu'aux crevasses de ce paysage pelé qu'il avait lui-même fait naître.

Dans «Les îles Mergui», c'est le Moi lui-même qui réfute sa propre construction métaphorique au profit d'une plongée plus essentielle dans un autre espace imaginatif. La première strophe est constituée de la 'lecture' d'une carte représentant l'archipel des Mergui, îles situées au sud de la Birmanie :

Les îles Mergui sont du frai.

Semence répandue entre les cuisses de la grenouille

Qui, Birmanie bleue, Siam jaune, Annam vert,

À croupetons rame, que le pied flottant de Malaka pousse

Dans les eaux chinoises ${ }^{17}$.

La poétisation mimétique ou métaphorique (les contours de l'archipel sur la carte sont comparés à ceux d'une grenouille et à du frai) est alors explicitement refusée :

Non.

Mes îles Mergui ne se baignent pas chantantes dans l'océan Indien ${ }^{18}$.

Une autre géographie se dessine alors, issue de la nuit et de l'eau : «D'une mer de nuit, taciturnes, elles émergent dans une aube qui jamais ne voit le jour $»^{19}$. S'ensuit la description de gigantesques buffles hirsutes fulminant dans une lumière déclinante, description qui dépasse la simple analogie de formes.

Le poème «L'Oural » présente une structure analogue : le point de départ du poème est une carte géographique, la première moitié de la première strophe évoque sur le mode imaginaire et fantastique la transformation des contours de l'Oural sur une carte en un dragon:

Voilà la montagne qui se dresse et se cabre comme la crête du dragon avec ses pics et ses crevasses.

Voilà l'Oural ${ }^{20}$.

Mais cette première approche ne suffit pas, la dimension graphique et la préhension par la main qui passe sur la carte sont refusées afin de faire véritablement advenir l'Oural :

Il me faut éteindre la lampe pour qu'il advienne, qu'il rampe devant moi, prodigieux lézard géant dans la nuit.

Car sa roche coule et sa forêt sort

De mon âme.

Et le souffle de ma bouche tisse des volutes au-dessus

Des neiges du Iaman-tau, mon sommet éternel.

Je médite ${ }^{21}$.

Ici aussi la représentation mimétique doit être dépassée pour que puisse se produire une fusion entre le Moi et l'espace entrevu par l'imagination. La strophe se termine, grâce à l'ambiguïté de l'expression "mon sommet éternel", par un acte d'appropriation (je possède désormais le Iaman-tau, massif dans lequel le fleuve Oural prend sa source) et/ou d'identification (je suis ce sommet). Le passage se fait par aveuglement au monde («il me faut éteindre la lampe », Kolmar utilise le même verbe « löschen » que dans « Art ») et entrée dans l'espace de l'intériorité : «je médite », qui a la même valeur structurale que la négation « Nein » dans « Les îles Mergui » et qui est aussi mis en relief typographiquement. 

monde extérieur :

Donne-moi ta main, ta chère main, et viens avec moi ;

Car nous voulons partir loin des hommes.

Ils sont mesquins et méchants, et leur mesquine méchanceté

nous hait et nous tourmente ${ }^{22}$.

Est opposée à la laideur du monde un locus amoenus peuplé d'animaux qui se trouvent au-delà du Bien et du Mal, dans une neutre animalité. L'évocation qui peut sembler 'naïve' de l'idylle imaginée doit être lue comme un contrepoint à un régime raciste qui s'emploie à établir une nomenclature délirante :

Cherchons donc refuge

Dans les champs rêveurs qui consolent gentiment la course de nos pieds avec des

fleurs et de l'herbe,

[...]

Auprès des animaux de la forêt qui ne médisent pas ${ }^{23}$.

Pourtant, dans ce recueil, la désignation d'un horizon utopique, loin de mettre un terme à l'écriture, n'en constitue qu'une première étape. Une lecture détaillée du poème «Les îles Mergui » permet de dégager les caractéristiques propres à la création des espaces imaginaires de Kolmar. Une fois rejetée la métaphorisation mimétique de l'archipel dessiné sur la carte, Kolmar décrit sa vision des îles, telles qu'elles surgissent d'une mer intérieure qui certes emprunte des traits à l'Océan indien, mais qui relève surtout d'une géographie imaginative qui trahit l'influence de lectures expressionnistes: les îles sont comparées à des buffles monstrueux dans un paysage glauque. La poétesse retrouve ici des procédés chers aux poètes expressionnistes tels que l'utilisation transitive de verbes intransitifs, les constructions anaphoriques et paratactiques («Leur naseau bouillonne l'écume/ leur flanc mugit l'obscurité $»^{24}$ ), l'utilisation de certaines couleurs destinées à créer une atmosphère (« vert tirant sur le noir ", "brunâtre ", " pâle » ${ }^{25}$ ). Au fur et à mesure que se déroule le poème, on entre dans un espace imaginaire peuplé d'oiseaux fabuleux inconnus des naturalistes, «des oiseaux sans essor recouverts de crins de cheval qu'aucun chercheur encore n'a identifiés $»^{26}$. Cet espace est surtout caractérisé par son statisme (les oiseaux sont expressément désignés comme dissociés d'une de leurs fonctions caractéristiques, le vol) ; il apparaît comme pétrifié. Ici la clairière est de pierre, l'œil du serpent est fixe, sa couleur elle-même évoque le schiste ( L'œil jaune du serpent gris ardoise, enroulé immobile, se lève vers l'éternel soir $\left.^{27} »\right)$; l'espace décrit est placé sous le signe du serpent, symbole de cycle et d'éternité, tout comme dans « Art » où le Moi poétique est accueilli par un personnage dont la couronne est entourée de deux basilics au regard pétrifiant. Le statisme de ce paysage lui confère une dimension éternelle explicitement nommée. Le paysage est également constitué de grottes calcaires rongées par les eaux. Elles ne sont pas peuplées d'hommes, mais de sauriens aquatiques fêtant d'étranges noces lubriques. Le lecteur, parvenu à cette strophe, a perdu de vue la forme des îles Mergui sur la carte de l'Extrême-Orient, il est dans un espace fantastique et archaïque qui est aussi sexualisé. C'est un univers où les règnes se mélangent: la végétation, la pierre, la faune échangent leurs qualités comme en témoignent le terme composé «Blumentiere» (textuellement «animaux-fleurs») qui désigne les coraux, ou l'expression «den pantherfleckigen porzellanen Mantel », « le manteau de porcelaine moucheté comme une panthère ${ }^{28}$. Cet univers, qui s'est creusé d'une strophe à l'autre, a gagné en outre en couleurs, la palette de Kolmar devient plus différenciée et plus 
contrastée (" vert malachite ", " rouge et bleu », « aux nageoires écarlates ", « sombres poitrines violettes ", " couleur œillet et safran $\left.{ }^{29}\right)$ : on a l'impression d'être dans un monde hybride entre règne végétal et règne minéral qui tient à la fois de Gustave Moreau et de Max Ernst.

La strophe suivante, par l'utilisation de formes du passé, semble vouloir expliquer comment l'homme a été expulsé de cet univers. Un naufrage (cataclysme naturel ou déluge divin) a détruit les traces de la culture, les œuvres des hommes. Art, agriculture, écriture, mécanisation, langage quotidien, argent ont disparu :

Des navires furent emportés par le vent.

Emportés...déchiquetés...Des planches, lambeaux du monde

Qui porte le poinçon de l'ouvrier et le crayon de l'écrivain

et la charrue, et le poids de la balance du marchand,

Mille roues pressées, mille paroles bredouillées

Et l'argent $[\ldots]^{30}$

L'énumération de ces «lambeaux du monde " trahit une critique de la modernité : y sont associées inflation monétaire et inflation verbale, mécanisation et aliénation. Par ailleurs, l'ordre dans lequel se fait cette énumération retrace l'histoire de l'évolution de l'humanité, de la civilisation agraire au capitalisme industriel moderne. L'univers qui résulte de ce cataclysme est un espace sans hymne au soleil ni élégie à la lune, un monde sans poète donc où un étrange "rat de grotte " (J. Lajarrige propose "protée " pour le terme "Grottenratte») couve un œuf turquoise tacheté de gris: ailleurs constitué d'une grotte, d'une caverne grotesque, où les propriétés dormitives de la belladone n'ont pas de sens en l'absence d'humains susceptibles d'en profiter. Cette peinture culmine dans une caractérisation qui concentre la nature de l'utopie de G. Kolmar : «Calme. Être, encore sans Faire $»^{31}$.

Cette plongée dans l'univers archaïque d'une nuit « inconstellée » est retour à l'Être, avant tout Faire. Ce qui dans l'ordre du texte apparaît comme intervenant après le naufrage des œuvres humaines, est une modalité de l'Être d'avant les œuvres. L'imagination poétique a construit un univers où règnent le silence et le calme, non pas une "vie antérieure" où comme chez Baudelaire "tout n'est que luxe, calme et volupté ", mais un état (bouddhique, mystique) d'être absolu. De façon caractéristique, le Moi s'est effacé, il n'y a plus que des formes substantivées : « Stille », « Sein », « ohne Tun.».

Cette descente dans l'archaïque, dans la pénombre des grottes marines ne s'arrête pas devant l'Être pur. Le poème comporte une dernière strophe qui présente un autre espace, sauvage, luxuriant, dont on ne sait pas où il se situe exactement par rapport au monde 'grotesque' du rat laiteux. Ce qui frappe, c'est que le calme qui confine au statisme est rompu par un acte sexuel :

Là où l'entrelacs de ramages étrangle de squameux troncs nains dans l'étreinte de

leurs maigres bras,

Sous le plumage de l'acacia,

Le fruit solitaire perce le calice vert profond,

Long et arrondi, renflement oblique de la pulpeuse rougeur

dénudée.

Il attend

Que le souffle léger et chaud de lèvres

Murmurantes tâtonne dans les fourrés, le touche, frémisse,

l'enveloppe : 
Et les fibres cachées dans la chair du fruit déversent

une semence féconde ${ }^{32}$. première strophe: «zeugenden Samen» ("semence féconde») fait écho à « hingesamt» («semence répandue »). Est-ce à dire que le mouvement imaginatif qui avait atteint la zone de l'Être, du Non-Agir est renvoyé à l'ordre mimétique, ou bien au contraire le renvoi n'est-il là que pour mieux marquer précisément la distance avec la lecture de la représentation cartographique ? La structure du poème fortement articulé autour du «Nein» donne à penser que c'est la deuxième interprétation qui est pertinente. Ce qui se produit au terme de ce poème, c'est une autre pro-création des îles Mergui selon les modalités propres au Moi lyrique ( $"$ mes îles Mergui ne se baignent pas chantantes dans l'Océan Indien »). Comme le dit Regina Nörtemann :

Dans le poème «Les îles Mergui », l'élément féminin, en quête d'un élément masculin en position d'attente, l'enveloppe pour concevoir ses propres «îles Mergui ", ces mêmes îles qui, avant cet acte de procréation, viennent d'être décrites avec précision et sur lesquelles finalement il a lieu. Le poème se génère pour ainsi dire lui-même dans un processus autoréférentiel ${ }^{33}$.

On peut préciser que l'isotopie de la semence n'est pas là pour rabattre le poème sur la première strophe, mais pour proposer une autre création dans un autre espace que celui de la carte étalée devant le sujet poétique. Dans la première strophe, le frai est simplement jeté devant ou entre les cuisses de la grenouille, dans la dernière strophe on trouve la description très précise d'une fécondation.

Une dialectique semblable est à l'œuvre dans «L'Oural » : la construction d'un ailleurs caractérisé par une beauté non humaine n'arrive toutefois pas à se stabiliser et ouvre sur des espaces inquiétants. Après avoir éteint la lumière et être entré par un acte de participation magique dans l'espace intérieur/extérieur de l'Oural, le sujet déclenche le processus imaginatif: le bestiaire de Kolmar, fait de loups, d'ours et de martres, s'anime. Il est frappant de constater que le Moi se dissocie pour mieux s'intégrer à l'espace qui est issu de lui. Au début du poème, il apparaît explicitement en tant que créateur, mais du fait de sa position et de sa participation au 'récit', il devient aussi personnage de l'espace créé : il a basculé de l'extérieur vers l'intérieur. Puis, de façon caractéristique, cet espace se creuse, à partir de l'évocation de racines, le regard imaginatif pénètre dans la dimension chtonienne de l'Oural fantasmé pour découvrir une caverne fabuleuse. Le paysage qui se déploie est de nouveau un espace pétrifié et scintillant où les règnes minéral, végétal et animal se confondent. Pierres, fruits, feuillage, coquillages sont reliés à la fois par des procédés métaphoriques et par le travail du signifiant: il est question d' "amandes de pierre » (« Mandelsteine ») qui mûrissent, image rendue possible par le double sens de "Stein» qui désigne la «pierre » mais aussi le «noyau» d'un fruit. Tel le Créateur qui, au sixième jour, constate que "tout est bon ", le sujet imaginatif peut déclarer, une fois que la grotte souterraine a été décrite : «Tout cela est beau »" La mise en relief typographique du vers souligne la dissociation du Moi qui est à la fois, dans l'espace et hors de l'espace, en position de sujet voyant mais à côté des objets vus: le sujet agit, crée, puis s'appréhende lui-même comme créature. Sur le modèle biblique, mais sous une forme inversée, le récit de la création n'est pas achevé, ce monde souterrain caractérisé par la beauté n'est pas complet.

Le poème qui promettait dans son titre et à la fin de la première strophe une évocation de l'Oural se poursuit par la description d'un autre monde qui, comme dans la dernière 
strophe des « îles Mergui », n'est pas situé par rapport à ce qui précède. Ici comme dans d'autres poèmes l'intériorité du Moi est spatialisée, mais l'espace qui en résulte n'est pas unique, il est fait de compartiments juxtaposés : «Mais j'ai autre chose encore ${ }^{35}$. La plongée dans l'espace imaginaire de l'Oural se transforme en introspection du Moi qui explore les deux dimensions de son être. Démiurge d'un univers post-symboliste paré de l'éclat des pierres précieuses et du prestige de leurs noms ${ }^{36}$, le Moi ne peut se défaire de cet autre côté situé en lui d'où s'échappe un être inachevé qui suscite son angoisse : « Des gouffres ombragés, où se tapit un monstre, demi-être qui m'a échappé avant que je ne lui provoque un arrêt cardiaque $»^{37}$.

31 La description d'un monde beau a basculé dans l'évocation d'un univers traumatique, il est suggéré que le Moi créateur veut ou doit se sacrifier : «Alors je m'avancerai/ Et les mains sur ma poitrine tremblante, je me pencherai au-dessus de l'abîme... $»^{38}$. C'est qu'il s'agit de délivrer cet être qu'il a voulu tuer, ou peut-être auquel il a voulu donner vie, car l'expression utilisée par Kolmar est volontairement ambiguë: de ce demi-être, organisme à moitié achevé il est dit qu'il a échappé au Moi, mais on peut aussi comprendre qu'il en est issu (le verbe "entschlüpfen» a deux, voire trois sens: " échapper » à l'emprise de quelqu'un, mais aussi " échapper » dans le sens d'un mot prononcé involontairement; enfin ce verbe, dans un sens cru, évoque l'expulsion hors du corps maternel). Par ailleurs, ce que le sujet a voulu lui donner, c'est un "Herzschlag" qui peut signifier "attaque cardiaque", mais aussi «battement de cœur ", respiration, vie. On songe ici au traumatisme qui a marqué la vie de Kolmar, à l'avortement auquel elle a été vraisemblablement contrainte sous la pression familiale à l'âge de vingt ans : le motif de l'enfant qui n'est pas né et de la nécessaire expiation traverse son œuvre, dans le cycle Mondes il se trouve au centre du poème « Sans fruit » 39 .

L'imagination poétique de Kolmar est frappée de mélancolie, les mondes qu'elle crée ne juxtaposent pas seulement des espaces beaux, idylliques et des univers inquiétants selon un principe de bipartition de l'espace tel qu'on le retrouve par exemple dans la tradition du conte. Au fil du poème se construit une utopie dont la beauté s'avère mortifère, et donc incapable de constituer un idéal praticable par le sujet poétique. Tout fonctionne comme si on était en présence d'un « autre » de l'utopie, un ailleurs de l'ailleurs qui vient jeter son ombre sur le lieu premièrement construit.

33 Ce décentrement de la perspective apparaît clairement dans un des poèmes les plus importants du recueil, «L'Ange dans la forêt ». Le titre promet l'évocation d'une figure typique de la situation de seuil : l'ange représente l'ailleurs en tant qu'il se manifeste aux humains, il appartient par définition à un espace intermédiaire. L'horizon d'attente induit par les éléments du titre correspond à cette fonction: le lecteur attend la relation d'une expérience de la transcendance dans l'immanence, d'un ailleurs céleste logé ici-bas, hors de la ville, dans la forêt. L'analyse du poème et en particulier du rapport à l'espace montre qu'en fait l'utopie est repoussée au fur et à mesure que le paysage se construit. Après avoir fui les humains et leur haine, le couple se retrouve dans un espace automnal rougeoyant, accueilli par un écureuil bondissant, "rouge flamme tremblante $\aleph^{40}$. Cependant, à peine nommé, le paysage automnal et sa mousse aux reflets d'émeraudes disparaît dans l'obscurité grandissante, il ne se constitue pas en refuge pour les amants en fuite. L'horizon utopique se déplace vers l'espace voûté de la nuit qui conformément à la tradition romantique semble seul pouvoir offrir un 
refuge. Par la succession des verbes au futur, puis immédiatement après au présent, Kolmar rattrape cette ligne d'horizon spatiale et temporelle :

Et voilà que s'ouvre une voûte

C'est sous son arcade bleu-gris entre les colonnes

Couronnées des arbres que se tiendra l'ange,

Grand et mince, sans ailes.

Son visage est souffrance ${ }^{41}$.

Cet ange est en rupture avec de nombreuses représentations de la figure angélique dans la tradition des différentes religions : il est chez Kolmar la figure paradoxale d'une souffrance impassible, voire indifférente. Il n'est pas cette figure rilkéenne du Terrible, ce «pollen de la divinité en fleur ${ }^{42}$ dont nous ne saurions supporter la vue. Il n'est pas poussé par le vent de l'Histoire, entrant à reculons dans le futur, les yeux braqués sur les ruines du passé comme l'Ange de Paul Klee dans l'interprétation de Walter Benjamin. Il est statique, muet, il n'est porteur de nulle Annonciation, de nulle consolation:

Et son habit a la pâleur glaciale des étoiles scintillant

dans les nuits d'hiver.

L'Étant,

Qui ne parle pas, ne doit pas, qui seulement est,

Qui ne connaît aucune malédiction ni bénédiction et qui

ne va pas en pèlerinage dans les villes rejoindre ce qui meurt ${ }^{43}$.

Dans les descriptions ou représentations iconographiques des religions monothéistes, les ailes que porte l'ange symbolisent sa promptitude et sa fonction de médiateur entre le ciel et la terre, la matière et l'esprit ${ }^{44}$. L'absence d'ailes de l'Ange de Kolmar au contraire nous invite à voir en lui une figure de l'immanence. Au milieu des fûts de cette forêt romantique qui fait jouer l'analogie entre nature et temple ou cathédrale, se tient un ange dont il est dit qu'il est l'Étant, manifestation de l'Être, et qui est à rapprocher du calme silencieux régnant au cœur des îles Mergui. Il est insensible au passage du temps (la feuille d'automne glisse sur lui), mais aussi aux humains :

Il ne nous regarde pas

Dans son silence argentin.

Mais nous, nous le regardons

Parce que nous sommes deux et abandonnés ${ }^{45}$.

Le regard n'est pas réciproque, il semble être l'apanage des humains qui vivent dans la scission et la déréliction ${ }^{46}$; lui qui ne va pas « dans les villes rejoindre ce qui meurt » est plus proche de l'ange de la deuxième "Élégie de Duino ", refermé sur lui-même, ou du Bouddha évoqué par Rilke dans les Nouveaux Poèmes :

ô, il est tout. Vraiment, attendons-nous de lui

Qu'il nous regarde ? Devrait-il en avoir besoin?

[...]

Lui qui oublie ce que nous connaissons

Et qui connaît ce qui nous rejette ${ }^{47}$.

Il n'est donc plus de séjour possible auprès de l'Ange, comme le titre du poème semblait le suggérer. Les deux personnages continuent leur route, ne pouvant trouver non plus dans la maison du père un abri, celle-ci, sombre et délabrée, étant «désormais la maison des orphelins qu'habitent des étrangers $\aleph^{48}$. Seul l'amant semble pouvoir constituer un refuge: la spatialisation du $\mathrm{Tu}$ interpellé constitue l'ultime ailleurs imaginé dans ce poème, un ailleurs intime placé sous le signe de l'amour mais aussi de l'impossible idylle. Les dernières lignes du poème représentent comme l'exact envers 
du locus amoenus évoqué dans la première strophe, les « champs rêveurs » qui devaient consoler «la course de nos pieds avec des fleurs et de l'herbe » font place à une vie d'exil sur les routes du monde qui s'achèvera de façon pitoyable. La rencontre avec l'Ange n'empêche pas le devenir-paria du sujet :

Tu es ma maison sur toutes les routes du monde [...]

Toiture tu es, exténué avec moi dans la fournaise

tu frissonneras quand fouettera la tempête de neige.

Nous aurons soif et faim, ensemble nous souffrirons,

Ensemble un jour sur le bord poussiéreux du chemin nous sombrerons et

pleurerons... ${ }^{49}$

En 1937, soumise de plus en plus aux vexations antisémites, Kolmar s'imagine, dans le poème "Asie ", fille d'exil rentrant chez elle auprès de sa mère, l'Asie, ce continent qu'elle ne connait pas et qui se distingue des autres en ce qu'il est, sans participer au faire :

Différente...

$\mathrm{Tu}$ as encore cette infinie patience muette,

La connaissance du Non-Agir, du calme immense qui est

absorbé par ses rêves,

Tienne est la vue,

Le regard mystérieusement levé dans la nuit bleue vers des mondes

lumineusement changeants.

Tu es comme si tu étais sans effets ${ }^{50}$.

Dans le poème qui met en scène ce retour, la Terre promise des ancêtres n'est évoquée qu'à la marge, dans un sens poétique ou biblique ${ }^{51}$ qui exclut toute interprétation sioniste, de même que dans le poème intitulé "Servir » le souvenir du pays des ancêtres était ravivé non pas dans une perspective eschatologique ou politique, mais dans le cadre plus banal de la préparation d'un plat assaisonné à l'huile d'olive (M 36-37). Il n'y a pas de rêve d'alya chez Kolmar. Le sujet contemple en imagination un monde, une Asie allégorisée et monumentalisée ( Ta fière stature voilée qui se dresse majestueuse sur le trône mythique/... Le front, mur nourrissant de grandes pensées $\aleph^{52}$ ) qui ellemême lève les yeux vers d'autres mondes lumineux. L'ailleurs chez Kolmar est ainsi mis en abyme dans un espace poétique qui fait jouer extériorité et intériorité, vision du Moi imaginatif et regard du monde imaginé sur d'autres ailleurs. Cet enchâssement d'espaces semble cependant figurer l'impossibilité d'un séjour, que ce soit dans la demeure du père («L'Ange dans la forêt ») ou au pied du trône de la Mère. Il est significatif que Kolmar place au centre du cycle un poème qui dénonce l'aliénation de la Mère Asie, dépossédée d'elle-même par son double, par son reflet, lui aussi allégorisé et anthropomorphisé, qui commerce avec les colonisateurs anglais :

$\mathrm{Au}$-dehors ton propre reflet mendie, prend et rafle,

ombre

Qui échangea les soieries suaves comme le crocus et l'orchidée, contre de l'étoffe anglaise, hideusement noire $[. . .]^{53}$

Le poème, censé décrire le retour du sujet exilé dans sa patrie se termine par une allusion au culte du feu dans la religion zoroastrienne et aux fameux dakhmas, ces " tours du silence » où les Parsis déposent leurs morts, laissant aux vautours le soin de débarrasser les ossements de la matière impure :

Quand tu es plongée dans les profondes entrailles de notre astre,

le bain de feu écumant...

Consume-toi...

Cache pleine de pudeur ce que l'insensé [le double de l'Asie] 
dénude, le secret

de ton cœur qui reçut la semence enflammée,

Et fais éternellement tournoyer les nouveaux-nés, démons

charognards,

au-dessus des tours mortuaires,

Tours du silence.... ${ }^{54}$

41

L'image utopique du retour auprès de la Mère, Reine installée sur son "trône mythique ", débouche sur un double mouvement où l'Asie est invitée à se retirer dans son secret et à abandonner les hommes à la malédiction du cycle des naissances et de la réincarnation.

En 1937 Gertrud Kolmar ne pouvait pas se douter qu'un tout autre feu allait s'abattre sur l'Europe et que seraient érigées de tout autres «tours du silence »; toutefois sa réinterprétation du culte parsi dans le sens d'une assimilation des vivants aux vautours nécrophages témoigne aussi, au cœur même de ce panorama de mondes intérieurs, de la vérité historique de sa poésie

\section{NOTES}

1. Traduction L.C. : «Ich will nichts mehr für mich. Ich will zugrunde gehen/ Zugrund - das heißt zum Meer, dort find ich Böhmen wieder./ Zugrund gerichtet, wach ich ruhig auf./ Von Grund auf weiß ich jetzt, und ich bin unverloren./ Kommt her, ihr Böhmen, alle, Seefahrer, Hafenhuren und Schiffe/ Unverankert. Wollt ihr nicht Böhmisch sein, Illyrer, Veroneser/ Und Venediger alle. Spielt die Komödien, die lachen machen/ Und die zum Weinen sind [...]», I. Bachmann : Werke, München/ Zürich, Piper, 1978, vol. I, p. 167-168.

2. J'utilise l'édition bilingue établie, postfacée et traduite par Jacques Lajarrige: Mondes, Paris, Seghers, 2001 (abrégée désormais en $\mathrm{M}$, suivi du numéro de la page). Elle est la première à présenter les poèmes du cycle dans l'ordre voulu par G. Kolmar. Sur les problèmes que pose l'édition de Hermann Kasack (G. K. : Das lyrische Werk, München, Kösel Verlag, 1960, reprise dans G. K. : Welten, Frankfurt a. M., Suhrkamp, 1999) voir plus bas. La récente édition de Regina Nörtemann (G. K. : Das lyrische Werk. 1927-1937, Göttingen, Wallstein) respecte, elle aussi, l'ordre du tapuscrit original.

3. «Irgendwo in Böhmen senkt eine Birke schmale blond/ Umflossene Wangen rötlichen Trümmern zu./ [...]/ Aus gierig glänzenden Augen der blauen Haie die spähend, schnappend in Küstengewässern sich tummeln,/ Blicken die Herren der Feste, Seelen illyrischer Seeräuber her/ [...]/ O schwarze Flaggen, Kaperfahrten, waffenschlagende/ Plankenkämpfe mit den Venedigern! » (M 42-45).

4. «Ich grenz noch an ein Wort und an ein anders Land/ Ich grenz wie wenig auch an alles immer mehr,/ Ein Böhme, ein Vagant, der nichts hat, der nichts hält/ begabt nur noch, vom Meer, das strittig ist, Land meiner Wahl zu sehen. », I. Bachmann : Werke, op. cit., vol. I, p. 168.

5. «Vorbei/ Aus den verfallenden Kammern/ Läuten nicht tausend goldene Becher mehr, die blut-/ Farben Weines voll sind [...]» (M 44-45).

6. « Aber die Söhne/ erben der Väter Gift. » (M 45). 
7. «Am Strande nördlichen Meeres/ Wo schwarzer grausamer Sturm Schwärme gell/ kreischender Möwen peitscht [...] Starrt der Turm./ Hart, finster, lastend, stumm in grauer Öde. » (M 42-43).

8. «Unsäglich leerer Himmel, der Vogelfittich und fruchtende/ Wolke nicht kennt, gießt brennende Bläue endlos/ über ihn aus;/ Gluthitzen, Strahlenfluten rinnen von seinen bleichen Quadern. » (M 44- 45).

9. «Vielleicht vergaß mich meine Seele im Traum,/ Sank gen Morgen gebreitet, und ihres Wandelfluges/ Harrte der weiße Turm: durch seine heißen,/ verwunschenen, lebenlosen Gemächer irrte sie,/ Ihre Ahnen suchend,/ Und rührte verschwebend Saiten an, die noch tönen... » (M 46-47).

10. «Vorüber das Einhorn, vorüber./ Denn seine leichten, flüchtigen Füße kamen weither/ aus dem Goldlande Ophir,/ [...]/ Doch das steile Horn seiner Stirnmitte goß sanfteres Licht, milchig schimmerndes,/ über die nackten Hände und weich umschleierten Brüste/ der Frau [...]» (M 28-29).

11. «Es sah/Einsame Städte, bleich, mit Kuppel und Minarett und den Steinen der Leichenfelder/Schweigend unter dem klingenden Monde. Es sah/ Trümmer, verlassene Stätten, nur von Geistern behaust,/ in funkelnder Finsternis/ Unter kalten Gestirnen./ Einmal lockte der Wüstenkauz,/ Und im Fernen heulten Schakale klagend ;/ Hyäne lachten. » (M 28-29).

12. «Formstrukturen als Sinnstrukturen. Der Zyklus als poetische Form im Werk Gertrud Kolmars ", in : Jacques Lajarrige (Hg.) : Vom Gedicht zum Zyklus. Vom Zyklus zum Werk. Strategien der Kontinuität in der modernen und zeitgenössischen Lyrik, Innsbruck, Studien Verlag, 2000, p. 98-121.

13. «Und die Wolke, die ihre zeichnende Hand geweht,/ Senkte sich und umwallte, hob und trug sie empor/ Zum Schrund ihrer kahlen Berge.// Ein Wartender,/ Dem zwei grüngoldene Basilisken den Kronreif schlangen,/ Stand im Dämmer auf, glomm und neigte sich, sie zu/ grüßen. » (M 136-137).

14. «Sie nahm den Silberstift/ Und hieß ihn hingehn über die weiße matt glänzende Fläche :/ Ihr Land. » (M 134-135).

15. «Farbenlos, wesenlos ist dies, ohne Stimme ; es redet zu uns nicht./ Kommt weiter. » (ibid.).

16. « Nur ihre Schulter zuckte, ihr Blick losch in Tränen. » (M 137).

17. «Die Mergui-Inseln sind Laich./ Hingesamt vor den Schenkeln des Frosches,/ Der, blaues Birma, gelbes Siam, grünes Annam,/ Hockt und rudert, den Schwimmfuß Malakka in chinesiche Fluten stößt. » (M 10-11).

18. « Nein.// Meine Mergui-Inseln baden nicht singend im indischen Meere. » (ibid.).

19. «Sie tauchen aus Nachtsee schweigsam in stetig tagloses Dämmer empor [...]» (ibid.).

20. «Da ist Gebirg, das mit Zacken und Schründen sich/ aufsteilt und bäumt wie eines Drachen Kamm./ Da ist der Ural. » (M 120-121).

21. «Ich muß die Lampe löschen, daß er werde, daß er vor mir/ krieche, riesiges Echsengetüm, in Nacht./ Denn es quillt sein Gestein, und sein Gewälde wächst/ Aus meiner Seele./ Und der Hauch meines Mundes webt rauchig über dem Schnee des Jaman-tau, meines ewigen Gipfels.// Ich sinne. » (ibid.).

22. «Gib mir deine Hand, die liebe Hand, und komm mit mir,/ Denn wir wollen hinweggehen von den Menschen./ Sie sind klein und böse, und ihre kleine Bosheit haßt und peinigt uns. » (M 58-59).

23. «So laß uns fliehen/ $\mathrm{Zu}$ den sinnenden Feldern, die freundlich mit Blumen und/ Gras unsere wandernden Füße trösten/ [...]/ Zu den Tieren des Waldes, die nicht übelreden. » (M 58-59).

24. «Ihre Nüster kocht Schaum/ Ihre Flanke rauscht Finsternis.» (M 10-11); traduction légèrement modifiée: Jacques Lajarrige propose "bouillonne d'écume", on peut comprendre aussi «kochen» dans le sens transitif de "produire une écume bouillonnante», le naseau devient ainsi actif.

25. « schwarzgrün », « bräunlich », «fahl », etc. (ibid.). 
26. « [...] mit Pferdshaaren, fluglose Vögel [...], die noch kein Forscher erkannt hat. » (M 12-13). 27. «Starrt mondgoldnes Auge schiefergrauer reglos/ gewundener Schlange in ewigen Abend auf. » (ibid.).

28. Ibid.

29. «malachitgrün», «blaurot», «scharlachflossig », «mit veilchendüsteren Brüsten », «nelken-und safranfarb» (ibid.).

30. «Schiffe wurden verweht. Verweht... Zerrissen... Planken treiben, Fetzen der Welt,/ Die den Meißel des Werkers trägt und des Schreibenden Stift/ und den Pflug und Kaufmanns Gewicht und Waage,/ Tausend hastende Räder, tausend haspelnde Worte/ Und das Geld. » (M 12-13).

31. La majuscule au verbe substantivé « faire » est de moi («Stille./ Sein noch ohne Tun. », 14).

32. «Wo Schlinggerank klammernd mit mageren Armen/ schuppige Zwergstämme würgt,/ Unter Akaziengefieder/ Bricht aus tiefgrüner Blattscheide einsame Frucht hervor,/ Lang und gerundet, steil in nackter, fleischiger Röte/ schwellend./ Sie wartet,/ Bis Lippen leisen, schwüleren Hauches/ Flüsternd durch Dickicht tasten, rühren, schauern,/ umhüllen:/ Sie bebet,/ Und die im Fruchtfleisch verborgenen Stränge gießen/ zeugenden Samen aus.» (M 14-15).

33. «In dem Gedicht Mergui-Inseln kommt das suchende Weibliche zum wartenden Männlichen, um - indem es sich über das Männliche stülpt - seine eigenen « Mergui-Inseln » zu empfangen, die vor diesem Zeugungsvorgang genau beschrieben werden und auf denen schließlich dieser Zeugungsvorgang stattfindet. Das Gedicht bringt sich sozusagen in einem selbstreferentiellen Prozeß hervor. » (R. Nörtemann : Gertrud Kolmar. op. cit., p. 391).

34. « All das ist schön». (M 122).

35. "Aber ich habe anderes noch... » (ibid.).

36. Kolmar aime les noms de pierres et des pierres précieuses, qu'elle utilise notamment pour désigner des valeurs de couleurs. On trouve entre autres mentionnés dans Mondes l'albâtre, le marbre, le granit, l'ardoise, la chrysolithe, la cornaline, la topaze, l'améthyste, la malachite, l'obsidienne, le jade, la tourmaline, l'émeraude, le saphir, etc.

37. «Schattenschlünde, da Ungestalt hockt, Halbwesen, das mir/ entschlüpfte, eh ich ihm Herzschlag gab. » (M 122-123).

38. «Dann werde ich hingehen/ Und, meine Hände auf bebender Brust, mich dem/ Abgrund neigen... » (ibid.).

39. M 113-117. Pour plus de détails sur les circonstances, en partie obscures, de ce traumatisme qui a conduit à une tentative de suicide, voir la biographie de Johanna Woltmann : Gertrud Kolmar. Leben und Werk, Frankfurt a. M., Suhrkamp, 2001, p. 86-92.

40. " rote zitternde Flamme» (M 58-59).

41. «Nun tut sich ein Gewölbe auf./ Unter seinem graublauen Bogen zwischen bekrönten/ Säulen der Bäume wird der Engel stehn,/ Hoch und schmal, ohne Schwingen./ Sein Antlitz ist Leid. » (M 60-61).

42. R.M. Rilke : CEuvres Poétiques et Théâtrales, dir. G. Stieg, Paris, Gallimard, 1997, p. 530 (trad. J.-P. Lefebvre) ; «Pollen der blühenden Gottheit » (R.M. Rilke : Sämtliche Werke, Frankfurt a. M., Insel, 1987, vol. I, p. 689).

43. « Und sein Gewand hat die Bleiche eisig blinkender Sterne/ in Winternächten./ Der Seiende,/ Der nicht sagt, nicht soll, er nur ist,/ Der keinen Fluch weiß noch Segen bringt und nicht in/ Städte hinwallt zu dem, was stirbt. » (M 60-61).

44. Dans la tradition juive, l'ange (mal'ach) porte des ailes, dans la tradition musulmane également le malâ'ika peut prendre figure humaine et être ailé.

45. «Er schaut uns nicht/ In seinem silbernen Schweigen./ Wir aber schauen ihn,/ Weil wir zu zweit und verlassen sind. » (M 60-61).

46. Il existe un autre poème qui porte le même titre « Der Engel im Walde » et qui date de 1933. Ici aussi l'Ange est déjà défini par l'Être : «Il ne faisait rien, il ne disait rien; il était » (« Er 
schaffte nichts, er sagte nichts; er war ", G. Kolmar: Das lyrische Werk, hrsg. von Regina Nörtemann, op. cit., p. 385). Il est difficile de partager l'interprétation de Heidy Margit Müller qui voit dans ce poème de 1933 « du point de vue mytho-poiëtique la rencontre du Moi avec la Mort qui est conjurée ici sous les traits d'une figure de lumière messianique, douce et silencieuse » ( “'Der Engel im Walde' entwirft mythopoetisch eine Begegnung des Ich mit dem Tod, der hier als eine messianische, sanfte, stille Lichtgestalt beschworen wird.», in "Paradigmatische Todesmythen in der Lyrik Gertrud Kolmars", dans Chryssoula Kambas (Hg.) : Lyrische Bildnisse. Beiträge zu Dichtung und Biographie von Gertrud Kolmar, Bielefeld, Aisthesis, 1998, p. 55-68, ici p. 64). Seule la souffrance de son visage pourrait permettre de rapprocher l'Ange du Christ, mais dans les deux versions son indifférence aux hommes et à leur mort en fait difficilement une figure messianique.

47. Trad. L.C. (pour les besoins de mon commentaire, je n'ai pas repris la traduction de D. Iehl, in : R.M. Rilke, Euvres Poétiques..., op. cit., p. 373) ; «O er ist Alles. Wirklich, warten wir,/ daß er uns sähe ? Sollte er bedürfen? [...] Er, der vergißt was wir erfahren/ und der erfährt was uns verweist », R.M. Rilke, Sämtliche Werke, op. cit., vol. I, p. 496.

48. « Aber jetzt ist es das Haus der Waise, und fremde Leute wohnen darin. » (M 60-61).

49. « Du bist mein Haus an allen Straßen der Welt, in jeder/ Senke, auf jedem Hügel./ Du Dach, $\mathrm{du}$ wirst ermattet mit mir unter glühendem/ Mittag lechzen, mit mir erschauern, wenn/ Schneesturm peitscht./ Wir werden düstern und hungern, zusammen erdulden,/ Zusammen einst am staubigen Wegesrande sinken und weinen... » (M 62-63).

50. Traduction modidiée : «Anders.../ Du hast noch die stumme unendliche Geduld,/ Das Wissen vom Nicht-Tun, gewaltiger Ruhe, die in sich/ versunken träumt, / Dein ist die Schau,/ Der rätselnde Aufblick in blaue Nacht zu leuchtend/ wandelnden Welten./ Du bist, ob du nicht wirkst. » (M 76-77).

51. Le Moi demande à l'Asie de bercer son oreille du chant des chalumeaux que pratiquait autrefois David à Beth-Lechem (M 76-77).

52. «Den stolzen verhüllten Wuchs, mächtig ragend von mythischem Throne [...]» (M 74-75).

53. «Draußen bettelt und nimmt und rafft dein eignes Abbild,/ Schemen,/ Der Seiden, lieblich wie Krokus und Orchidee, mit häßlichem englischen Tuch vertauschte [...]» (M 78-79).

54. «Wenn du hinabgetaucht zum tiefen Innen unseres Sterns,/ dem Bade schäumenden Feuers.../ Brenne.../ Birg voll Scham, was die Törichte blößt, deiner Mitte/ Geheimnis, das Flammensamen empfing,/ Und die Geborenen, Geierdämonen, laß ewiglich kreisen,/ über den Totentürmen,/ Türmen des Schweigens... » (M 78-79).

\section{RÉSUMÉS}

En 1937, six ans avant sa mort à Auschwitz, Gertrud Kolmar écrivit à Berlin un cycle de 17 poèmes intitulé Mondes (Welten). Ces univers imaginaires dans lesquels on ne trouve pas de références explicites à la persécution nazie sont situés pour la plupart dans un 'ailleurs' à la fois spatial et temporel, occidental et 'oriental', intérieur et extérieur. Peut-on dire pour autant que Kolmar 'se réfugie' dans un univers irréel de création littéraire? Si le sujet aime se présenter comme entrant dans l'espace même qu'il a construit, cet espace est toutefois marqué par la mélancolie ou l'angoisse, de sorte que s'ouvre souvent un deuxième espace, un 'ailleurs de l'ailleurs' qui n'est pas moins ambigu. On est en quelque sorte en présence d'un «Autre » de l'utopie qui vient jeter son ombre sur le lieu premièrement construit. Les mondes imaginés, à la beauté de pierre mais 
désertés par les humains, peuvent certes faire signe vers l'Être pur mais sont également associés à la mort ou à la disparition du sujet. L'écriture apparaît non pas comme une simple conduite de fuite devant les événements contemporains, mais comme l'exploration d'un espace littéraire qui est aussi un 'ailleurs' intérieur ou extérieur dans lequel le sujet affronte sa propre mort.

1937, sechs Jahre vor ihrem Tod in Auschwitz, schrieb Gertrud Kolmar einen aus 17 Gedichten bestehenden, Welten betitelten Zyklus. Die Mehrzahl dieser imaginären Landschaften, in denen nicht explizit auf die NS-Verfolgungen verwiesen wird, befindet sich in einem zugleich räumlichen und zeitlichen, westlichen und östlichen, inneren und äußeren 'Anderswo'. Kann man aber sagen, Kolmar würde in unwirkliche erdichtete Welten flüchten? Zwar wird das lyrische Ich gern als ein schaffendes dargestellt, das dann in den von ihm geschaffenen Raum eintritt; dieser Raum ist jedoch durch Melancholie bzw. Angst geprägt, so dass sich oft ein zweiter Raum eröffnet, der aber wiederum nicht weniger zweideutig ist. Man steht sozusagen vor dem 'Anderen der Utopie', das seinen Schatten auf die primär erfundene Landschaft wirft. Die imaginären, ineinander verschachtelten Räume mit ihrer erstarrten und menschenleeren Schönheit können zwar auf das reine Sein hindeuten, sie werden aber auch mit dem Tod oder dem Verschwinden des Subjekts verbunden. Schreiben erweist sich demnach nicht als bloße Flucht vor dem Zeitgeschehen, sondern als Erkundung eines inneren bzw. äußeren 'Anderswo', in dem das Subjekt sich seinem Tod stellt.

In 1937, six years before her death in Auschwitz, Gertrud Kolmar wrote in Berlin a cycle of 17 poems, untitled Worlds (Welten). These imaginary universes in which no explicit reference to Nazi persecution can be found, are located for most of them in a "distance" meanwhile spatial and temporal, western and "eastern", inner and outer. It is necessarily possible to say that Kolmar 'shelters' in an unreal universe of literary creation? If the subject likes presenting itself as entering in the very space he has built, this space is yet marked par melancholia or anxiety; so that a second space, "an elsewhere of an elsewhere", not less ambiguous, often open. We are in a way, in presence of an "Other" of utopia that comes or cast a shadow on the place firstly built. The imagined worlds, with stone beauty but deserted by human beings, can indeed make a sign to the pure Being but are also associated with death and loss of the subject. The writing appears not as a simple conduct of escape before the contemporary events but as the exploration of a literary space that is also an inner or outer "elsewhere" where the subject faces his own death.

INDEX

oeuvrecitee Mondes, Welten

\section{AUTEURS}

\section{LAURENT CASSAGNAU}

École Normale Supérieure-Lettres Sciences Humaines (Lyon) 